\title{
BMJ Open Protocol for an exploration of knowledge sharing for improved discharge from a mental health ward
}

\author{
Emma Rowley, ${ }^{1}$ Nicola Wright, ${ }^{2}$ Justin Waring, ${ }^{1}$ Kyri Gregoriou, ${ }^{3}$ Arun Chopra ${ }^{3}$
}

To cite: Rowley $\mathrm{E}$, Wright $\mathrm{N}$, Waring J, et al. Protocol for an exploration of knowledge sharing for improved discharge from a mental health ward. BMJ Open 2014:4:e005176

doi:10.1136/bmjopen-2014005176

- Prepublication history for this paper is available online. To view these files please visit the journal online (http://dx.doi.org/10.1136/ bmjopen-2014-005176)

Received 3 March 2014 Revised 21 August 2014 Accepted 26 August 2014

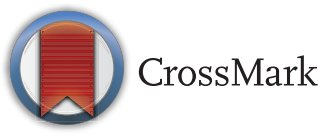

${ }^{1}$ Centre for Health Innovation, Leadership \& Learning, Nottingham University Business School, Nottingham, UK ${ }^{2}$ School of Health Sciences, University of Nottingham, Nottingham, UK

${ }^{3}$ Adult Mental Health Services, Nottinghamshire Healthcare NHS Trust, Nottingham, UK

Correspondence to Dr Emma Rowley; emma.rowley@nottingham. ac.uk

\section{ABSTRACT}

Introduction: Strategies to reduce hospital admissions for mental health service users have received vast amounts of attention, yet the transfer of care from hospital to the community has been ignored. The discharge process is complex, messy, disjointed and inefficient, relying on cross-agency and organisational working. Focusing on one acute mental health admission ward, we will investigate whether the discharge process for people with severe mental health problems can be enhanced through the creation, implementation and utilisation of a knowledge sharing proforma that is used on their admission to the ward. Methods and analysis: The project uses qualitative interviews to understand the complex processes associated with being admitted and discharged from inpatient mental health wards. Practitioners will be asked to identify and map the relevant stakeholders involved in admission and discharge, and discuss any problems with the process. The study team will work with clinicians to develop a knowledge collection proforma, which will be piloted for 2 months. Qualitative interviews will be carried out to collect reflections on the experiences of using the tool, with data used for further refinement of the intervention. Baseline and repeat quantitative measures will be taken to illustrate any changes to length of stay and readmission rates achieved as a result of the study.

Ethics and dissemination: A key issue is that participants are able to comment frankly on something that is a core part of their work, without fear or reprise. It is equally important that all participants are offered the opportunity to develop and coproduce the knowledge collection proforma, in order that the intervention produced is fit for purpose and usable in the real world, away from a research environment. The study has received ethical approval from Nottingham University Business School ethics committee, and has all appropriate National Health Service research governance clearances.

\section{INTRODUCTION}

The Care Quality Commission, the UK's healthcare regulator, has recently suggested that the lack of psychiatric inpatient beds is causing stress to services and patients. ${ }^{1}$

\section{Strengths and limitations of this study}

- Applied health research-takes complex social theory ideas and applies them to an area of healthcare that is often ignored (mental health).

- Study and intervention is coproduced with end-users.

- Exploratory study - data collection on only one ward.

- Quantitative measures likely to be influenced by a complex healthcare context.

At the same time, there is growing evidence that approved mental health professionals are detaining people under a section, illegally, in order to obtain a bed. ${ }^{23}$

In the UK, $10 \%$ of psychiatric beds $(1700$ beds) have been cut since $2011 .{ }^{4}$ Conversely, the numbers of people detained under the Mental Health Act reached a record high in 2011/12 with 48600 people being detained, a $5 \%$ rise on $2010 / 11$ levels. ${ }^{4}$

Many National Health Service (NHS) Mental Health Trusts have adopted functional splits to inpatient and outpatient care, whereby different teams lead care and treatment with an individual at different phases of their illness. However, rather than continue to work in these operational silos, inpatient and outpatient teams need to seamlessly interact with the admitted service user to develop a single narrative and purpose to the admission, while also participating fully in the process of discharge. We believe that there is scope to improve this practice, with the increased efficiency in knowledge sharing leading to timelier, safer and higher quality discharges.

Strategies to reduce hospital admissions and to help mental health service users remain in the community have received the attention of researchers. Studies have explored the efficacy of crisis care planning, ${ }^{5}$ recovery planning ${ }^{6}$ and the effectiveness of service delivery models such as Assertive 
Outreach. ${ }^{7}$ However, the same cannot be said for the transfer of care from hospital back to home, or from hospital-based to community-based care. In the East Midlands (UK), about $10 \%$ of patients are readmitted within a month of discharge, although this figure varies between different wards. There is no published national data on readmission rates. Readmission rates act as a proxy measure, albeit a crude one, for failed discharge. There has been no study looking at the factors that are associated with higher readmission rates.

We completed a literature review using search terms: mental health, discharge, adult (aged 18-65 years), acute and inpatient, using the ASSIA, CINAHL, EMBASE, MEDLINE and PSYCHINFO electronic databases. This returned only 139 citations, of which just six full-text empirical papers were obtained. To be included in our review, papers needed to be published since 2000. This was justified on the basis that studies needed to be relevant to current mental healthcare provision. In total, 139 citations were returned, and following the removal of duplicates, non-empirical literature and studies not conducted within mental health services, six full-text papers were obtained.

Of the papers included, two were from the UK, one each from Australia, Canada, Germany and the USA. Owing to the heterogeneity in the study designs (one systematic literature review, one qualitative study, one retrospective case note analysis and three surveys) a narrative approach to the synthesis of the identified literature was adopted and the key areas highlighted were:

- The handover of information between professionals. ${ }^{8}$

- Facilitative discharge approaches. ${ }^{9}$

- The challenges of delayed discharge. ${ }^{10} 11$

- Discharge planning interventions specifically in relation to outpatient follow-up appointments. ${ }^{12}$

- The use of an inpatient keyworker and peer support worker to assist service users with the transition from hospital to the community. ${ }^{13}$

Despite the different methods employed, these studies have highlighted some useful findings. Regardless of the service and organisational variations across the different countries these papers originated from, the problems and difficulties encountered in sharing information between professionals working in inpatient and community settings was consistent. For example, Durbin et al describe the quality of information sharing and reporting between primary care and mental health services that takes place at referral and postdischarge as, at best, variable. However, the use of interventions, such as liaison services ${ }^{12}$ and specific workers to assist service users with the transition from hospital to community, were found to produce improvements ${ }^{8} 912$ and therefore demonstrate that this process is amenable to intervention. The issue of 'delayed discharge' at an organisational level was explored by the two UK-based studies. ${ }^{10}{ }^{11}$ Although they both highlight that there are differences in the reporting and definition of 'delayed discharges' across the UK, delayed discharges remain a concern with potential financial ramifications. Lewis and Glasby ${ }^{11}$ suggest that organisations are desperate to tackle delayed discharges by any means possible. This includes supporting policy directives, such as reimbursement, when in other circumstances they would not do so.

Although these studies have highlighted some interesting findings, the lack of a robust evidence base indicates a need for further research into the transfer of care process, particularly as a 'critical period' of postdischarge care (the first 7 days), when people with mental health problems are at increased risk of suicide, has been identified. ${ }^{14}$ Suicide is a devastating consequence for the individual, their families and mental health professionals, but it is also relatively rare. In contrast, a range of more frequent and 'mundane' care problems often arise from care transition planning, which impact the costs and quality of life for people with mental health problems and their carers. Although there is a lack of evidence exploring these problems, anecdotal reports highlight difficulties such as medication not being available for service users on their return to the community, community nurses and social workers not being aware that an individual has been discharged, and disruption in social security benefits leaving service users without an income and financially dependent on others. In relation to delayed discharge from hospital, each additional day on the ward incurs a cost in excess of $£ 340,{ }^{15}$ while the Care Services Partnership and the National Institute for Mental Health in England identify the following 'human' consequences:

- Stressed, bored and anxious inpatients.

- Owing to increased lengths of time other service users wait for therapeutic intervention and arrangement of care packages.

- Overstretched and insufficient staff.

- An increased risk of serious incidents, substance misuse, self-harm, violence and aggression on the wards.

- Potential delays in admitting appropriate at risk service users or the premature discharge of others.

- Inappropriate transfer of service users between wards and services.

- An increased risk of service user dependence on inpatient care and subsequent loss of coping skills postdischarge.

- The loss of community contacts and supports such as friends, tenancies and employment.

- A negative impact on staff morale, retention and recruitment. ${ }^{16}$

Research by Waring et $a l^{17}$ shows that discharge planning and the transition of care is located within complex systems of interacting and interdependent actors. Strategies to coordinate the work of heterogeneous actors and mitigate system complexity are increasingly recognised within the social science literature, ${ }^{18}$ but have not been applied to the problems of hospital discharge for people with mental health problems. In particular, the social science literature highlights the 
importance of knowledge sharing as a basis of collaboration and coordination. ${ }^{19-22}$

Sullivan and Williams suggest that "the health, social care and well-being needs of vulnerable people are complex and inter-related. They require carefully planned, coordinated and delivered interventions from a number of different professional groups working together", 23 yet healthcare delivery is increasingly categorised by its fragmented, multiprofessional teams and partnerships that cross organisational boundaries and, as such, 'the provision of seamless healthcare and social care remains problematic'. ${ }^{23}$

A recent (unpublished) audit in the NHS Trust where this research is to take place has shown that the admission and discharge process is complex, multifaceted and involves a significant number of healthcare practitioners from across a variety of different occupations and agencies. Significantly, although all these practitioners hold knowledge about the patient's transition of care, there is no central knowledge repository where all this information is being collated in a manner that allows for its readily available access and utility. Although the Trust has recently introduced electronic patient records, the number of computers on the ward is limited, which means timely and immediate access to records (as well as updating them) can be problematic. This results in information being variously recorded in different sections of the patient's notes, where each clinical grouping makes notes in 'their' section, often without crossreferral to other sections. This information is then later transposed onto the electronic health record, often by ward administrative staff.

A further consequence of the difficulty in accessing electronic health records in a timely manner is that apart from the paper-based patient files, the other main source of information collected and used are personal notes often carried around by the individual practitioner, for example, those taken during the nursing handover or when taking a telephone call about an incoming patient admission. This has led to an inconsistent information collection process, where gaps in knowledge about service users have resulted. Such information deficits have led to practitioners repeatedly collecting the same information as their colleagues and thus duplicating work. Moreover, the gaps in knowledge about the patient, which need to be addressed in order to plan a safe and effective discharge, are often not identified in a timely enough manner, and are instead only being flagged once discharge is imminent. We therefore suggest that the problem is one of knowledge sharingand, in particular, the breakdown in sharing knowledge and the resultant gaps in knowledge which appear.

Public policies advocate collaborative partnerships to foster more inclusive and 'joined-up' service delivery mechanisms. ${ }^{24}$ This is largely premised on improved knowledge sharing, whereby actors are able to communicate information across occupational, organisational and sectoral boundaries, and meet a mutual set of objectives, which should ultimately result in a more streamlined and integrated way of working. ${ }^{25}$ Knowledge sharing can represent a powerful source of service integration, efficiency and, importantly, safety. However, there are major challenges to this; communication 'breakdowns' represent a major barrier to service efficiency and safety; NHS 'collaboratives' and 'mandated networks' are bedevilled by professional cleavages and power differentials that inhibit knowledge sharing. ${ }^{25} 26$

There is growing evidence of the social and organisational processes involved in care transitions, including the importance of communication, yet this rarely takes account of the complex social and cultural dynamics of knowledge sharing. The literature on knowledge sharing relates, more broadly, to theories and concepts associated with interpersonal and occupational communication; knowledge exchange and brokering; translational research; and organisational learning. This diverse literature shows that various interpersonal, social and organisational factors influence knowledge sharing and learning within complex organisations, including the appreciation of distinct knowledge domains, social hierarchy, accessibility, and psychological safety and trust. ${ }^{27}$ Knowledge is shown to be both 'slippery', where it is too difficult to codify, as well as 'sticky' or difficult to share across cultural or institutional boundaries. ${ }^{28} 29$ Such research also highlights the various strategies for facilitating knowledge sharing, such as 'knowledge brokers' who can translate and transfer knowledge between isolated groups, information and communication technology to provide easy access and retrieval to knowledge and 'communities of practice' that engender cultural and organisational alignment through knowledge sharing. ${ }^{30} 31$

Given the clinical risks associated with hospital discharge, it continues to be a national policy priority, ${ }^{32}$ with the advice that care transitions should be seen as 'a process not an isolated event' ${ }^{33}$ involving the active participation of healthcare and social care professionals, as well as service users and carers, to effectively plan and coordinate discharge. This whole system approach highlights the interdependency of individuals and organisations from different care delivery settings. However, the most common threats to timely and efficient hospital discharge are associated with notifying and organising 'external services'. ${ }^{34}$ This highlights the importance of communication between care providers, yet the literature on hospital discharge offers little in way of this, especially in relation to discharge from acute mental health services. As highlighted previously, our literature review identified only one study that explicitly explored information and communication provision in relation to discharge between primary care providers and inpatient services in the USA.

It is important to understand the barriers and drivers to a patient's care transition not as linear casual chains within single or isolated care settings, but as complex and enmeshed 'constellations' of factors found within and between care processes and teams. This includes 
the deeper 'darksides' of service organisation and delivery, ${ }^{35}$ such as organisational boundaries and the shifting of responsibility and endemic problems of interprofessional and interorganisational working, which typically relate to problems in communication or knowledge sharing. ${ }^{36}$ Glasby $^{37}$ suggests three prominent factors influence the participation and coordination of these different stakeholders, which are also consistent with the whole systems and systems thinking approaches. These include: (1) occupational factors, related to the particular knowledge, culture and practice domains of care providers, such as doctors, social workers and nurses; (2) organisational factors, related to the routine working patterns, facilities, capacities and resources of individuals agencies and (3) compatibility and coordinating factors, related to how occupational, organisational and institutional factors align, including communication, decisionmaking and resources.

Consequently, in piecing together the jigsaw of contemporary, complex, integrated healthcare, individual practitioners and healthcare workers must mediate boundaries to their knowledge sharing, which act to decipher what constitutes the expert and legitimate participation of particular groups of people in particular circumstances. ${ }^{38}$ These boundaries can be "physical, cognitive, relational, structural, knowledge based or any other delineation that separates one boundary from another" ${ }^{39}$

The resulting gaps have been described as structural holes, fissures and silos; ${ }^{40}$ they act to "shine a light on how communication breaks down, interactivity fails or where teamwork is weak or floundering. Structural holes are often at the boundaries of organisational silos and this can enable and impede interprofessional relations or interunit knowledge transmission". ${ }^{40}$ Boundaries or silos between different professions and professional practices have long been recognised (eg, medical tribalism; ${ }^{23}{ }^{41}$ they are known to inhibit knowledge sharing, ${ }^{36}$ to the extent that they are 'a significant brake on quality improvement initiatives'). ${ }^{42}$

Crossing boundaries and connecting separate work and knowledge domains requires coordination for effective knowledge sharing to occur. Boundary crossing describes the actions and activities of a person, a group or an intervention that makes 'transactions and interactions' across different sites. ${ }^{38}$ Boundary crossing is a 'challenge of negotiating and combining ingredients from different contexts to achieve hybrid solutions', ${ }^{43}$ and is a means of acquiring and controlling knowledge. ${ }^{17}$ Thus for activities that require linking or brokering across and between boundaries, there is a need to search for connections in order to mobilise and share knowledge across the professional territories, and create links to avoid fragmentation, disconnection and, ultimately, to prevent patient need from being left unaddressed.

Swan and Scarborough ${ }^{44}$ call for dedicated knowledge brokering roles, arguing that these enable 'the transfer of knowledge across organisational and interorganisational boundaries'. Braithwaite $e t a l^{45}$ develop this line of argument further, referring to the dissemination of information via 'grapevines', which interweave between individuals who are linked through a common purpose. Effective and timely communication, for instance, between hospital and the Community Mental Health Team (CMHT) is essential in ensuring appropriate transition from the hospital into the community. Yet, it is hypothesised that in the acute inpatient mental health experience, given the complexity and interagency working that occurs, there is no one or nothing carrying out this brokering role across the boundaries and being the central information repository resource.

In other healthcare sectors, it is possible that the patient would be an ideal candidate to act as a knowledge broker and facilitate the sharing of knowledge and information about their care requirements and medical history to different practitioners-as it is the patient who is the constant across the various healthcare and social care interactions that take place. However, service users being admitted to an acute mental health ward, often without their explicit consent (ie, they are on a section of the Mental Health Act), are quite likely to lack the capacity and ability to act in this knowledge broker role at the moment of their admission to the ward. What is more, as their care will have been delivered by multiple providers and agencies, as well as family members and significant others, there is not a central knowledge repository that can be drawn on. Rather, information presented on admission to the ward can be sketchy and incomplete, with practitioners and administrators searching for information from multiple sources. This is not just a waste of valuable resources, but also delays the admission procedure and, in turn, failure to identify complete knowledge about the patient can delay their treatment and eventual discharge from the ward.

We suggest that knowledge sharing between the service user (where possible), professionals and carers during the admission and discharge planning processes can speed up the process and reduce the knowledge gaps that are known to create delays and blockages to discharge. We will focus on one acute mental health admission ward to investigate how the discharge planning and transition process can be enhanced, in terms of making discharge more effective through improved knowledge sharing. Knowledge sharing requires collaboration and coordination in order to be effective, ${ }^{19-22}$ as planned rather than ad hoc actions are required in order to address the difficulties in information sharing in fragmented care settings. ${ }^{23}$ As we have already described, staff on the ward are known to keep 'personal notes' containing information about a patient. Through the development, implementation and utilisation of a knowledge collection proforma that will be completed by healthcare staff on the service user's arrival to the ward, we will seek to formalise these personal notes, so that they are stored in a patient's (paper-based) notes folder rather than remaining in the pocket of a healthcare practitioner. This study is the first step towards the 
production of a shared knowledge collection resource, which can be used by all healthcare and social care practitioners involved in the admission and discharge of patients from an acute mental health ward. If this is shown to be effective, further funding will be sought to develop and roll out an electronic version.

\section{METHODS AND ANALYSIS}

Service users will be involved at every stage of the planning and management of the study. We will convene a small group of approximately five members who have had recent experience of being admitted and discharged from hospital. This group will be facilitated by a member of the research team and will meet approximately bimonthly. They will discuss the planning and development of the project and intervention, be involved in data analysis and disseminate the study findings to service user forums and in service user focused publications. They will also be invited to be involved in the development of any subsequent research grant applications and follow-on studies should these occur. If any members of the service user group wish to join the full study management team, they will be enabled to do this; otherwise their views and work will be relayed to the full team by the individual who facilitates the group. Service users will be paid a 'disruption' fee to cover their time and travel costs incurred through being involved in the study.

This study follows an improvement science approach. Although a relatively new term, which is often interchangeably used along with translational science, implementation science, evidence-based practice, knowledge translation and research utilisation, the overarching goal of improvement science is to ensure that quality improvement efforts are evidence based. ${ }^{46}$ Improvement science offers a rigorous yet practical approach to understanding and implementing quality improvement, as it "inhabits the sphere between research and quality improvement by applying research methods to help understand what impacts on quality improvement". ${ }^{47}$ As such, it "focuses on systematically and rigorously exploring 'what works' to improve quality in healthcare and the best ways to measure and disseminate this to ensure positive change". ${ }^{47}$

The study will address the following research question:

How can inappropriate discharge and subsequent readmission be reduced through greater knowledge sharing during inpatient admission and discharge planning?

Secondary objectives are:

1. To explore the knowledge sharing process and procedure on admission to an adult acute mental health ward.

2. To seek to improve knowledge sharing to prevent delayed discharge because of information gaps.

Qualitative data collected will seek to understand the complex processes and blockages occurring during knowledge sharing linked to a patient's admission and discharge from the acute ward. This will then be used by the research team to produce a knowledge collection proforma, which will aim to prompt healthcare practitioners to be aware of knowledge gaps in the patient's history, and raise questions/take action where this is needed.

Using a mixed-methods approach, an in-depth understanding of the complex processes associated with being admitted and discharged from inpatient mental health wards will be ascertained. Healthcare practitioners' (acute and community-based) perceptions of the appropriateness of this new knowledge collection tool will be evaluated using qualitative methods, and will be supplemented by quantitative data analysis, through baseline and repeat measures of anonymised patient length of stay and readmission rates.

Anonymised baseline data collected at the start of the study will be repeated at the completion of the pilot, to measure any change that has occurred in average length of stay and in patient readmission rates as well. Readmission is defined locally as "patient readmitted within a month of initial discharge'. Length of stay and readmission rate measures have been selected as they are both aligned to a CQUIN (Commissioning for Quality and Innovation) target (21 days median length of stay) and are requested by the local Clinical Commissioning Groups to inform their decision-making.

The project is structured across a number of work packages. The initial phase of the project will identify the relevant stakeholders and information sources involved in mental health admission and discharge. Subsequent work will engage and work with clinicians and healthcare workers to develop the intervention (the knowledge capture proforma), which will be piloted in the latter phases of the project. Following this we will ask those involved to reflect on their experiences of using the proforma, so that this can be used to further develop the intervention and apply for further funds for a larger scale study.

- Work package 1 will see anonymised baseline data taken on the average length of stay on the study site (Ward A) and readmission rates over the last calendar year. The study team will carry out a series of qualitative interviews with clinicians and healthcare practitioners working in acute and community care settings who are involved in the admission and discharge processes of patients into/out of Ward A. Additionally, any admission and discharge packs, or other knowledge collation documents, will be collected and studied, to identify what information is currently assembled, by whom and when, where it is stored and when it is used.

- Work package 2 will draw on the learning from work package 1 , and will involve the production of a new knowledge capture proforma to be used at the time of the patient's admission to the acute ward. Following the initial analysis of the data from work 
package 1, the new proforma will be coproduced with members of the acute and community teams in a series of workshops. By co-designing the proforma in this manner-similar to a user-based design approach, it ensures that the resulting product is fit for use by the practitioners, as they have had a role in coproducing the outcome.

- Work package 3 will involve the roll out of the knowledge collection proforma produced in work package 2. Its introduction will be supported by a series of short presentations made by the study team to healthcare staff working in Ward A, as well as to those healthcare staff who are involved in the admission or discharge of patients from Ward A. The proforma will be piloted for two calendar months on Ward A.

- Work package 4 will comprise of a series of evaluative qualitative interviews with the clinicians and practitioners who will have been using the new knowledge capture proforma. These interviews will collect data on perceptions and experiences of using the proforma, and whether practitioners feel its implementation and use has enhanced the admission and discharge process through having access to more knowledge about the patient. Repeated baseline measures on anonymised length of stay and readmission rates will be taken, to cover the pilot dates, in order to provide quantitative evidence of any effect that the form may have had.

The study follows a Plan-Do-Study-Act cycle.

Work packages 1 and 2 fall under the Plan stage; work package 3 comprises of the Do stage; work package 4 fulfils the Study stage, while the final Act stage will be covered in a future funding application, to develop this pilot study further, and to cover its roll out and evaluation across the whole of the NHS Trust involved.

\section{Study configuration}

This is a single site study; the predominant focus is on a single acute mental health ward. However, in order to respond to the hypothesis and research objectives, it will also be necessary to include healthcare staff working in community care (employed by the same NHS Trust as the ward-based staff) who are involved in the referral and admission/discharge process to/from Ward A.

Ward A is a busy acute mental health ward in an urban setting within the UK. It has 20 beds for male patients. Patients typically have been diagnosed with schizophrenia, bipolar disorder, severe depression or borderline or antisocial personality disorder, often with comorbid substance misuse problems and sometimes with other physical health problems.

\section{Recruitment}

Participants for the study will all be employed by the NHS Trust, and either be working on Ward A or be a member of a community team (eg, Crisis Teams, Community Assessment and Treatment, Early Intervention in Psychosis, Recovery and Assertive
Outreach, CMHTs) that admits service users to the ward or is involved in their care following discharge. No service users or their carers/family members will be recruited to participate in the study at this stage. The initial approach will be from AC, who is a consultant on the ward, and will be made verbally and followed up in writing, accompanied by a participant information sheet and consent form.

\section{Sample size and justification}

Sample size is determined by the number of relevant stakeholders working in/into Ward A, rather than by power calculations or expectations about study dropout. The sample size will be approximately 50 healthcare practitioners. This number covers all those healthcare workers who would reasonably be expected to have some interaction with Ward A in relation to the admission or discharge of a patient.

\section{Eligibility criteria}

In order to be eligible to be involved in the study, participants should be employed by the NHS Trust and have a role in the admission and/or discharge of patients from Ward A.

Consequently, in order to be eligible to be included in the study, participants should be:

- Aged between 18 and 65 years.

- Working in the NHS.

- Involved in the admission and/or discharge of patients from Ward A

Conversely, individuals will be excluded from participating in the study if they are not employed by the NHS Trust; have no experience of admission/discharge of patients to/from Ward A, and are unable to give consent.

All participants will provide informed consent before being enrolled in the study.

\section{Data collection}

As illustrated in table 1, two sets of qualitative interviews will be held with all healthcare practitioners who interact with Ward A regarding the admission and discharge of patients; these are scheduled to take place during work packages 1 and 3. Qualitative interviews have been chosen as a data collection technique so as to enable the space for reflective reporting and open discussion of the phenomena under investigation. To this end, a topic guide will be utilised (see table 2 for an indicative illustration of the topics that might be covered). Interviews will be carried out by NW and ER, recorded with participants' consent, and transcribed verbatim.

Work package 2 involves a series of co-design workshops with the healthcare practitioners previously interviewed. These workshops will be practical in focus, and will seek to produce a knowledge capture proforma, which will ensure that knowledge regarding admission and discharge is shared between the different healthcare practitioners. Co-design is founded on the principle that "making it 'better' is possible if users are involved in the 
Table 1 Study regime

\begin{tabular}{|c|c|c|c|}
\hline & Timeline & Research team activities & Participant involvement \\
\hline 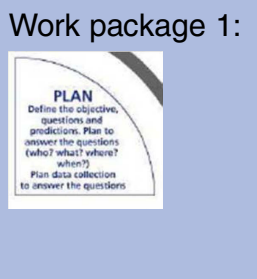 & $\begin{array}{l}\text { Late Autumn } \\
2013 \text {-January } \\
2014\end{array}$ & $\begin{array}{l}\text { Baseline data collection } \\
\text { Collection and analysis of } \\
\text { admission and discharge } \\
\text { documents } \\
\text { Analysis }\end{array}$ & $\begin{array}{l}\text { 1. Participants to be identified (by AC, acting } \\
\text { as gatekeeper to the clinical setting) } \\
\text { 2. Invitations issued, accompanied by } \\
\text { participant information sheet and consent } \\
\text { form) } \\
\text { 3. Interview scheduled } \\
\text { 4. Interview takes place }\end{array}$ \\
\hline 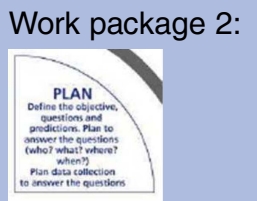 & $\begin{array}{l}\text { January-February } \\
2014\end{array}$ & - Analysis & $\begin{array}{l}\text { 1. Participants invited to participate in } \\
\text { coproduction/co-design workshops for } \\
\text { knowledge capture tool/proforma } \\
\text { 2. Workshops arranged and take place }\end{array}$ \\
\hline $\begin{array}{l}\text { Work package 3: } \\
\text { Do } \\
\text { corry out ene pen }\end{array}$ & March-April 2014 & $\begin{array}{l}\text { Roll out of the knowledge } \\
\text { capture tool, supported by } \\
\text { training presentations }\end{array}$ & \\
\hline 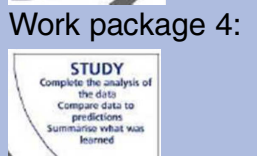 & $\begin{array}{l}\text { May-November } \\
2014\end{array}$ & $\begin{array}{l}\text { Repeat measures } \\
\text { Analysis } \\
\text { Reporting and dissemination }\end{array}$ & $\begin{array}{l}\text { 1. Staff interviews regarding their perceptions } \\
\text { and experiences of using the knowledge } \\
\text { capture tool. }\end{array}$ \\
\hline
\end{tabular}

design process". ${ }^{48}$ The approach, while practical, also enables discussion of "how well people understand (the intervention being designed), how they feel about it while they are using it, how well it serves its purpose, and how well it fits into the context in which they are using it". ${ }^{48}$ It is this 'knowledge of the experience' in relation to the problems and potential solutions to knowledge sharing and capture in relation to admission and discharge from the acute mental health ward, that is 'unique and precious'. 48

Marshall et al ${ }^{49}$ argue how "improvement science needs a genuine partnership between academics and front-line practitioners. Researchers bring scepticism, scientific rigour and methodological technical expertise, whereas practitioners bring content knowledge, a thorough understanding of working contexts, and practical wisdom. Academics and service partners need to collaborate to design, undertake and interpret the work of improvement science". Together, the two approaches of co-design and improvement science offer potential to produce a step change in knowledge sharing and reduce the delays to discharge caused by communication failure.

\section{Data analysis}

The interview data will be analysed using conventional qualitative methods, and will identify analytical patterns from across individual respondent and wider service. ${ }^{50} 51$ Analysis will be inductive, although it will be influenced by the study's theoretical framework of knowledge brokering and knowledge mobilisation. ${ }^{31} 52-54$

Table 2 Interview topic guides

\section{Topics to be covered in work package 1 interviews}

- Experiences of patient admission process to Ward A-what is involved, who is involved, how long does it take?

- Perceived problems with the patient admission process.

- How, when and by whom is knowledge and information gained, stored and shared?

- Experiences of patient discharge process from Ward A-what is involved, who is involved, how long does it take?

- Perceived problems with the patient admission process.

- How, when and by whom is knowledge and information gained, stored and shared?

- About delays in discharge-how often? Caused by what? What knock-on effects?

\section{Topics to be covered in work package 3 interviews}

- About using the new knowledge capture form

- Did knowledge sharing in relation to patient admission process to Ward A improve while the form was being used?

- Any problems with the form?

- How might it be improved?

- Having used the form, how, when and by whom is knowledge and information gained, stored and shared?

- Following using the form, what have been the experiences of the patient discharge process from Ward A-what is involved, who is involved, how long does it take?

- Have delays in discharge through gaps in knowledge about a patient's circumstances been reduced? 
Thematic analysis has been chosen as it "provides a concise, coherent, logical, non-repetitive and interesting account of the story the data tell" ${ }^{55}$ Although time intensive-it requires the research team to spend time engaging with the data, reading and rereading the interview transcriptions and listening to the audio recordings of interviews-we believe that it offers unparalleled advantages in 'getting to know' your data. In turn, this 'generates understanding, insight and familiarity, which are the building blocks of analysis'. ${ }^{55}$ The research team will start to identify and code (by highlighting) parts or chunks of the data that they deem to be about the same topic, concept or idea. It is likely that many sections of the data will be given multiple codes, implying that the section/extract is about more than one topic or idea. As codes are developed it is also important to revisit the rest of the data to see if a particular code also applies to other parts of the data. Initially, the data will be analysed separately by each member of the research team; following initial coding, the research team will hold regular data meetings in which they will work collaboratively on the analysis of the interview materials.

Owing to the need for the research team to analyse the data collaboratively, a CAQDAS (Computer Assisted Qualitative Data Analysis) package, NVivo, will be used. This will allow each member of the research team to add their own comments and analysis. The use of NVivo allows for sections of the transcript to be tagged, or highlighted and named with a certain code or label; these programmes do not undertake the analysis of the data for you, but they do allow the electronic data to be coded and searched, for notes to be written to accompany codes and data extracts, for the data to be more easily navigated and for the accumulating analytical work to be located on a single data corpus. While early reading and immersion in the data can be carried out using hard copies of the transcripts, once a number of initial codes have been generated, transcripts will be imported into NVivo, with all coding of the data undertaken on the electronic versions of the transcripts from then on.

Following this preliminary analysis stage, in which all the data extracts will have been coded in a general sense, data will again be examined in order to identify the wider themes and analytical narrative.

The quantitative data from the baseline and repeat measures (length of stay; readmission rates) will be analysed simply; given the complexity of the admission/discharge process, it will not be possible to statistically show the 'effect' of the proforma due to so many confounding influences. However, by running simple statistical tests (such as median length of stay and average readmission rates) over two time periods (before/during the use of the proforma), we may be able to see some difference, which would then suggest the need for more detailed, subsequent investigation if the research were to be repeated on a larger scale.

\section{ETHICS AND DISSEMINATION}

While the ethical issues faced in policy-oriented, qualitative research are not of the same order as those facing research involving invasive clinical interventions, this is not to say that they can be brushed aside. The ethical and design issues that are of particular importance in this kind of research relate to the need to recognise the ways in which the social relationships relating to the phenomena being studied may impact on the research process, by impeding some participants from fully expressing their views while encouraging others to do so.

A key issue is that participants in this research will be asked to comment frankly on something which is a core part of their work, as this relates to the actions of other individuals and organisations involved in knowledge sharing and brokering during the admission and discharge processes. From the point of view of us as researchers, of good research practice and of the participants themselves, it is clearly important that those involved are as frank as possible, so that we might get a clear picture of what has helped and obstructed the knowledge brokering during the admission and discharge process in the particular context of acute mental health. If some respondents are franker than others, we may get a skewed view, and of the role of different factors and individuals in the process. This quandary is amplified by the fact that there may well be entrenched power relationships within the groups of individuals being studied, with certain parties exerting considerably more influence than others, which may make those less influential parties more reluctant to be frank. For example, senior consultants are likely to be perceived by other participants as more powerful than a healthcare assistant or an occupational therapist. For this reason, we will carefully manage group dynamics during the co-design workshops, to ensure that all parties are able to equally and fully participate.

When discussing the research with participants at the recruitment stage, we will emphasise that the views of all involved are equally important, and that we will make every effort to use what they tell us in a nonattributable way.

\section{Dissemination}

Study results will be published and disseminated in a variety of ways. A report of the study will be produced, including an executive summary, which will be distributed to participants and any other interested party. Peer-reviewed publications in academic outlets will be pursued, as will outputs in practitioner-oriented publications. Participants will not be identified in any publications.

This study sets out to coproduce a solution to an enduring problem in healthcare practice. Knowledge sharing among different healthcare teams is neither a new phenomenon nor something that is recognised as easily solved. It is also something that many researchers 
before us have attempted to improve. However, our focus on knowledge sharing on admission and discharge from an acute mental health ward, informed by improvement science and co-design approaches, offers a potential solution that is locally produced and owned. We hope that this approach will offer sustained benefits to patients and healthcare practitioners.

Contributors ER led the development of the study protocol and this manuscript. NW, JW, KG and AC commented on drafts of the protocol and the manuscript. ER and NW led the data analysis.

Funding This work was supported by Nottingham University Business School's 'Spark' research fund.

Competing interests ER and JW are members of NIHR CLAHRC East Midlands and, as such, their involvement was funded by the NIHR. AC is the main consultant psychiatrist on the ward where the study will be conducted, and is the problem owner, having identified the issue requiring improvement and bringing it to other members of the research team. $K G$ is a senior nurse on the ward.

Ethics approval University of Nottingham Business School research ethics committee.

Provenance and peer review Not commissioned; externally peer reviewed.

Open Access This is an Open Access article distributed in accordance with the Creative Commons Attribution Non Commercial (CC BY-NC 4.0) license, which permits others to distribute, remix, adapt, build upon this work noncommercially, and license their derivative works on different terms, provided the original work is properly cited and the use is non-commercial. See: http:// creativecommons.org/licenses/by-nc/4.0/

\section{REFERENCES}

1. Commission CQ. Board Paper: thematic reviews-future topics and a proposals for a more systematic approach to topic selection. 2013

2. Chopra A. Are our mental health services in crisis? Nottinghamshire Fabian Society, 2013

3. Hudson J, Webber M. The National AMHP Survey 2012: final report Stress and the statutory role: is there a difference between professional groups? London: King's College London, 2012.

4. Centre HaSCl. Inpatients formally detained in hospitals under the Mental Health Act 1983, and patients subject to supervised community treatment, annual figures, England, 2011/12 2013.

5. Thornicroft G, Farrelly S, Szmukler G, et al. Clinical outcomes of Joint Crisis Plans to reduce compulsory treatment for people with psychosis: a randomised controlled trial. Lancet 2013;381:1634-41.

6. Cook JA, Copeland ME, Hamilton MM, et al. Initial outcomes of a mental illness self-management program based on wellness recovery action planning. Psychiatr Serv 2009;60:246-9.

7. Priebe S, Fakhoury W, Watts J, et al. Assertive outreach teams in London: patient characteristics and outcomes. Pan-London Assertive Outreach Study, part 3. Br J Psychiatry 2003;183:148-54.

8. Durbin J, Barnsley J, Finlayson B, et al. Quality of communication between primary health care and mental health care: an examination of referral and discharge letters. J Behav Health Ser $R$ 2012;39:445-61.

9. Jerpersen $\mathrm{S}$, Chang $\mathrm{T}$, Donegan $\mathrm{T}$, et al. Reflections on facilitated discharge from a mental health service. Australisian Psychiatry 2009;17:195-201.

10. Impey M, Milner E. Delayed discharge from mental health inpatient care in the UK. Ment Health Pract 2013;16:31-5.

11. Lewis R, Glasby J. Delayed discharge from mental health hospitals: results of an English postal survey. Health Soc Care Community 2006; $14: 225-30$.

12. Steffen $\mathrm{S}$, Kosters $\mathrm{M}$, Becker $\mathrm{T}$, et al. Discharge planning in mental health care: a systematic review of the literature. Acta Psychiatrica Scandinavica 2009;120:1-9.

13. Martin ML, Jensen E, Coatsworth-Puspoky R, et al. Integrating an evidence bassed research intervention in the discharge of mental health clients. Achives Psychiatr Nurs 2007;21:101-11.

14. Meehan J, Kapur N, Hunt IM, et al. Suicide in mental health in-patients and within 3 months of discharge. National clinical survey. Br J Psychiatry 2006;188:129-34.
15. Gainsbury S. Calculating the cost of mental health. Health Serv J 2012;122:10-11.

16. England CSINIfMHi. A positive outlook: a good practice toolkit to improve discharge from inpatient mental health care. 2007.

17. Waring J, Currie G, Crompton A, et al. An exploratory study of knowledge brokering in hospital settings: facilitating knowledge sharing and learning for patient safety? Soc Sci Med 2013;98:79-86.

18. Perrow C, Wilensky HL, Reiss AJ. Complex organizations: a critical essay. New York: McGraw-Hill, 1986.

19. Parent R, Roy M, St-Jeacques D. A systems-based dynamic knowledge transfer capacity model. J Knowledge Manag 2007;11:81-93.

20. Jensen CB. Sociology, systems and (patient) safety: knowledge translations in healthcare policy. Sociol Health IIIn 2008;30:309-24.

21. Ward V, Smith S, House A, et al. Exploring knowledge exchange: a useful framework for practice and policy. Soc Sci Med 2012;74:297-304.

22. Wilkinson H, Gallagher M, Smith M. A collaborative approach to defining the usefulness of impact: lessons from a knowledge exchange project involving academics and social work practitioners. Evid Policy 2012;8:311-27.

23. Sullivan H, Williams P. Exploring the role of objects in managing and meditating the boundaries of integration in health and social care. $J$ Health Organ Manag 2012;26:697-712.

24. Klijn E-H, Teisman GR. Institutional and strategic barriers to publicprivate partnership: an analysis of Dutch cases. Public Money Manag 2003;23:137-46.

25. Bate SP, Robert G. Knowledge management and communities of practice in the private sector: lessons for modernising the National Health Service in England and Wales. Public Adm 2002;80:643-63.

26. Addicott R, McGivern G, Ferlie E. Networks, organizational learning and knowledge management: NHS cancer networks. Public Money Manag 2006;26:87-94.

27. Cross R, Borgatti SP. The ties that share: relational characteristics that facilitate information seeking. In: Huysman $\mathrm{MH}$, Wulf $\mathrm{V}$, eds. Social capital and information technology. Cambridge: MIT Press, 2004:137-61.

28. Pritchard $\mathrm{C}$, Chumer $\mathrm{M}$, Willmott $\mathrm{H}$, et al. Managing knowledge: critical investigations of work and learning, New York: Palgrave Macmillan, 2000.

29. Scarborough $\mathrm{H}$, Swan J. Explaining the diffusion of knowledge management: the role of fashion. Br J Manag 2001;12:3-12.

30. Lave J. Situating learning in communities of practice. In: Resnick L, Levine J, Teasley S, eds. Perspectives on socially shared cognition. Washington, DC, USA: American Psychological Association, 1991:63-82.

31. Lomas J. The in-between world of knowledge brokering. BMJ 2007;334:129-32.

32. Audit Commission. The way to go home; rehabilitation and remedial services for older people. London, 2000.

33. Health Do. Discharge from hospital: pathway, process and practice. London: The Stationary Office, 2003.

34. Agency NPS. National reporting and learning system quarterly data summary-England, Issue 13. London: NPSA, 2009.

35. Vaughan D. The dark side of organizations: mistake, misconduct and disaster. Annu Rev Sociol 1999;25:271-305.

36. Currie G, Waring J, Finn R. The limits of knowledge management for UK public services modernization: the case of patient safety and service quality. Public Adm 2008;86:363-85.

37. Glasby J. Hospital discharge: integrating health and social care. Radcliffe Publishing, 2003.

38. Akkerman SF, Bakker A. Boundary crossing and boundary objects. Rev Educ Res 2011;81:132-69.

39. Chreim S, Langley A, Comeau-Vallee M, et al. Leadership as boundary work in healthcare teams. Leadership 2013;9:201-28.

40. Braithwaite J. Between-group behaviour in health care: gaps, edges, boundaries, disconnections, weak ties, spaces and holes. A systematic review. BMC Health Serv Res 2010;10:1-11.

41. Long JC, Cunningham FC, Braithwaite J. Bridges, brokers and boundary spanners in collaborative networks: a systematic review. BMC Health Serv Res 2013;13:1-13.

42. Powell AE, Davies HTO. The struggle to improve patient care in the face of professional boundaries. Soc Sci Med 2012;75:807-14.

43. Engeström Y, Engeström R, Kärkkäinen M. Polycontextuality and boundary crossing in expert cognition: learning and problem solving in complex work activities. Learn Instr 1995;5:319-36.

44. Swan J, Scarbrough $\mathrm{H}$. The politics of networked innovation. Hum Relations 2005;58:913-42.

45. Braithwaite J, Runciman WB, Merry AF. Towards safer, better healthcare: harnessing the natural properties of complex sociotechnical systems. Qual Saf Health Care 2009:18:37-41.

46. Shojania KG, Grimshaw JM. Evidence-based quality improvement: the state of the science. Health Aff 2005;24:138-50. 
47. Foundation TH. Improvement science: research scan. London: The Health Foundation, 2011.

48. Bate $\mathrm{P}$, Robert $\mathrm{G}$. Experience-based design: from redesigning the system around the patient to co-designing services with the patient. Qual Saf Health Care 2006;15:307-10.

49. Marshall M, Pronovost P, Dixon-Woods M. Promotion of improvement as a science. Lancet 2013;381:419-21.

50. Ritchie J, Spencer L. Qualitative data analysis for applied policy research. In: Bryman A, Burgess RG, eds. Analyzing qualitative data. London: Routledge, 1994:173-94.

51. Huberman A, Miles M. Data management and analysis methods. In: Denzin NK, Lincoln YS, eds. Handbook of Qualitative Research. Thousand Oaks: Sage Publications, 1998:428-44.
52. Carlile PR. A pragmatic view of knowledge and boundaries: boundary objects in new product development. Organ Sci 2002;13: $442-45$.

53. Davies HTO, Nutley SM, Walter I. Why 'knowledge transfer' is misconceived for applied social research. $J$ Health Serv Res Policy 2008;13:188-90.

54. Tetroe J, Graham ID. How to translate health research knowledge into effective healthcare action. Healthc Q 2007;10:20-2.

55. Rowley E. Protocol for a qualitative study exploring the roles of 'Diffusion Fellows' in bridging the research to practice gap in the Nottinghamshire, Derbyshire and Lincolnshire Collaboration for Leadership in Applied Health Research and Care (CLAHRC-NDL). BMJ 2012;2012:1-7. 